\title{
Laser spectroscopy of muonic hydrogen
}

\author{
Randolf Pohl ${ }^{1, *}$, Aldo Antognini, ${ }^{1,2}$, Fernando D. Amaro ${ }^{3}$, François Biraben ${ }^{4}$, João M. R. Cardoso ${ }^{3}$, \\ Daniel S. Covita ${ }^{3,5}$, Andreas Dax ${ }^{6}$, Satish Dhawan ${ }^{6}$, Marc Diepold ${ }^{7}$, Luis M. P. Fernandes 3 , Adolf Giesen 7 , \\ Andrea L. Gouvea ${ }^{3}$, Thomas Graf7, Theodor W. Hänsch ${ }^{1, * *}$, Paul Indelicato ${ }^{4}$, Lucile Julien ${ }^{4}$, \\ Cheng-Yang Kao ${ }^{8}$, Paul Knowles ${ }^{9}$, José A. M. Lopes ${ }^{3}$, Eric-Olivier Le Bigot ${ }^{4}$, Yi-Wei Liu ${ }^{8}$, Livia Ludhova ${ }^{9}$, \\ Cristina M. B. Monteiro ${ }^{3}$, Françoise Mulhauser ${ }^{1,9}$, Tobias Nebel ${ }^{1}$, François Nez ${ }^{4}$, Paul Rabinowitz ${ }^{10}$, \\ Joaquim M. F. dos Santos ${ }^{3}$, Lukas A. Schaller ${ }^{9}$, Karsten Schuhmann ${ }^{2,11}$, Catherine Schwob4, \\ David Taqqu ${ }^{12}$, João F. C. A. Veloso ${ }^{5}$, Jan Vogelsang ${ }^{1, * * *}$, and Franz Kottmann ${ }^{2}$
}

Received 15 March 2013, revised 26 April 2013, accepted 30 April 2013

Published online 3 June 2013

Muonic hydrogen $(\mu \mathrm{p})$ is a very sensitive probe of the proton structure. Laser spectroscopy of two $2 \mathrm{~S}-2 \mathrm{P}$ transitions in $\mu \mathrm{p}$ was used to determine both the Lamb shift and the hyperfine splitting of the $2 S$ state in $\mu \mathrm{p}$. The rms charge radius of the proton, $R_{c h}=0.84087(39) \mathrm{fm}$, was extracted from the Lamb shift. The Zemach radius of the proton, $R_{Z}=1.082$ (37) fm, was obtained from the 2 S-hyperfine splitting. This article summarizes the previously published findings.

\section{Introduction}

The exotic muonic hydrogen atom $(\mu \mathrm{p})$ is made from a proton and a negative muon. Due to its large mass $m_{\mu} \approx 200 m_{e}$, the muon's Bohr radius is nearly 200 times smaller than the corresponding Bohr radius in (regular) electronic hydrogen $(\mathrm{H})$, causing a $200^{3} \approx 10^{7}$ times increase in the muon's wavefunction overlap with the proton. Sensitivity to nuclear structure corrections is correspondingly increased making laser spectroscopy of $\mu \mathrm{p}$ energy levels a very sensitive probe of e.g. the charge and Zemach radii of the proton.

We have recently determined the $2 \mathrm{~S}-2 \mathrm{P}$ energy splitting (Lamb shift) and the 2S hyperfine splitting (HFS) in $\mu \mathrm{p}$ for the first time $[1,2]$ (see Fig. 1).

\section{Method}

We have built a novel beam line for negative muons at low-energy (3-6 keV). About $600 \mu^{-}$per second enter a
$200 \mathrm{~mm}$ long hydrogen gas target, filled with $1 \mathrm{hPa} \mathrm{H}_{2}$ gas at room temperature. Roughly half of the muons stop, in a $200 \mathrm{~mm}$ long stop volume, with width and height of $12 \mathrm{~mm}$ and $5 \mathrm{~mm}$, respectively.

Before entering the $\mathrm{H}_{2}$ target, the muons are individually detected in two sets of ultra-thin carbon foils. Electrons ejected from the foils create a signal in plastic scintillators read out by photomultiplier tubes [3]. With efficiencies of $80 \%$ and $71 \%$ for the two foil detectors, we obtain $330 \mathrm{~s}^{-1}$ coincidences, each indicating the arrival of a muon.

Muons slow down in the target gas and are eventually captured by an $\mathrm{H}_{2}$ molecule. The molecule breaks up and a muonic hydrogen atom $\mu \mathrm{p}$ is formed at a high principal

\footnotetext{
* Corresponding author E-mail: randolf.pohl@)mpq.mpg.de

** also at: Ludwig-Maximilians-University, Munich, Germany.

*** present address: Institut für Physik, Carl von Ossietzky Universität, Oldenburg, Germany.

1 Max-Planck-Institut für Quantenoptik, Garching, Germany

2 Institute for Particle Physics, ETH Zurich, Switzerland

3 Departamento de Física, Universidade de Coimbra, Portugal

4 Laboratoire Kastler Brossel, École Normale Supérieure, CNRS and Université P. et M. Curie, Paris, France

5 I3N, Departamento de Física, Universidade de Aveiro, Portugal

6 Physics Department, Yale University, New Haven, CT, USA

7 Institut für Strahlwerkzeuge, Universität Stuttgart, Germany

8 Physics Department, National Tsing Hua University, Hsinchu, Taiwan

9 Département de Physique, Université de Fribourg, Switzerland

10 Department of Chemistry, Princeton University, Princeton, NJ, USA

11 Dausinger \& Giesen GmbH, Stuttgart, Germany

12 Paul Scherrer Institute, Villigen, Switzerland
} 


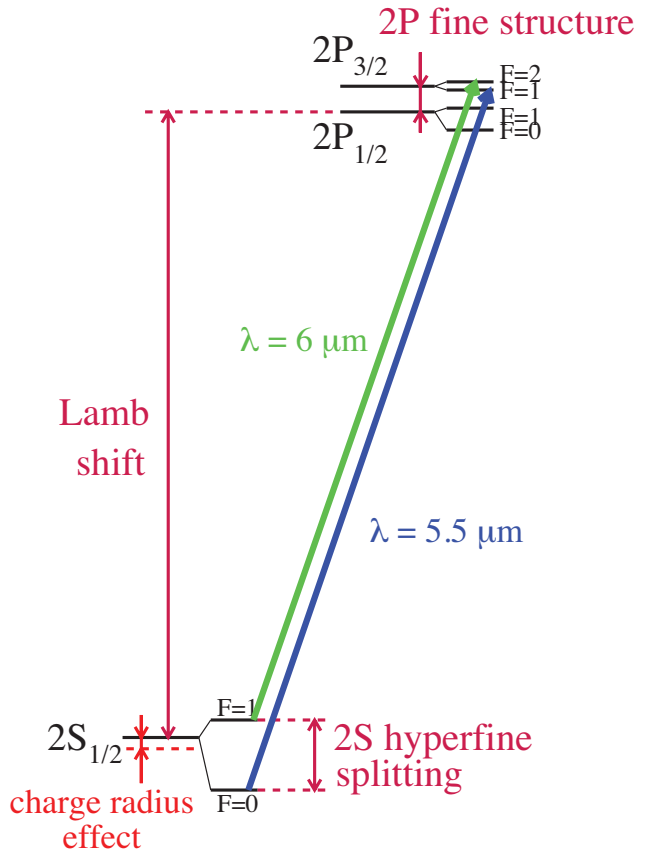

Figure 1 Level scheme of the $n=2$ states in $\mu \mathrm{p}$. The Lamb shift is dominated by vacuum polarization which shifts the $2 \mathrm{~S}$ state below the $2 \mathrm{P}_{1 / 2}$ state. There is a $2 \%$ contribution of the proton charge radius to the Lamb shift. The $2 S$ hyperfine splitting is affected by the Zemach radius of the proton (not shown).

quantum number $n \approx 14$. Several processes contribute to the deexcitation of the $\mu \mathrm{p}$ atom [4-7]. In the end, about $1 \%$ of the stopped muons form $\mu \mathrm{p}$ atoms in the metastable $2 S$ state, whereas the remaining $99 \%$ of the muons proceed to the 1S ground state via emission of Lyman x-rays $\left(\mathrm{K}_{\alpha}\right.$ at $1.9 \mathrm{keV}, \mathrm{K}_{\beta}$ at $2.2 \mathrm{keV}$, etc.) [8]. The lifetime of the $2 \mathrm{~S}$ state is about $1 \mu \mathrm{s}$ at $1 \mathrm{hPa} \mathrm{H}_{2}$ gas pressure, limited by collisional deexcitation $[9,10]$.

Above and below the muon stop volume, two rows of 10 large area avalanche photo diodes (LAAPDs, each with an active area of $14 \times 14 \mathrm{~mm}^{2}$ ) are mounted, at a distance of about $8 \mathrm{~mm}$ from the muon beam axis. The LAAPDs detect muonic K-x-rays with a time resolution of $35 \mathrm{~ns}$ and an energy resolution of 30\% (FWHM) [11].

An incoming muon triggers the laser system $[12,13]$. It provides $5 \mathrm{~ns}$ long pulses of $0.25 \mathrm{~mJ}$ energy, tunable from $5.5 \mu \mathrm{m}$ to $6 \mu \mathrm{m}$. A key requirement is a time delay of less than $1 \mu \mathrm{s}$ (dictated by the lifetime of the $\mu \mathrm{p}(2 \mathrm{~S})$ state) between the randomly occuring muon trigger and light output. This short delay is accomplished by a cwpumped, pulsed Yb:YAG oscillator producing pulses at $1030 \mathrm{~nm}$, only $200 \mathrm{~ns}$ after a trigger [13]. After amplification and second-harmonic generation, these pulses are used to pump a pulsed Ti:sapphire laser system which is seeded by a cw Ti:sapphire ring laser. The $15 \mathrm{~mJ}$ pulse of red light around $708 \mathrm{~nm}$ obtained from the Ti:sapphire laser system is then converted to the desired 5.5-6 $\mu \mathrm{m}$ infrared (IR) wavelength by three sequential Stokes shifts in a high-pressure $\mathrm{H}_{2}$ Raman cell [12]. The wavelength of the IR light is tunable by changing the wavelength of the cw Ti:sapphire laser. The IR wavelength is calibrated with an accuracy of $300 \mathrm{MHz}$ against well-known water vapor absorption lines.

A multipass mirror cavity inside the hydrogen gas target ensures homogeneous illumination of the muon stop volume. On resonance, laser-induced muonic hydrogen $2 \mathrm{~S}-2 \mathrm{P}$ transitions are signalled by the observation of $\mathrm{K}_{\alpha}$ $\mathrm{x}$-rays from the subsequent $2 \mathrm{P}-1 \mathrm{~S}$ deexcitation.

\section{Results}

The Lamb shift in $\mu \mathrm{p}$ was determined as 202.3706(23) meV, corresponding to a relative uncertainty $u_{r}=11 \mathrm{ppm}$. The 2S-HFS was found to be 22.8089(51) $\mathrm{meV}\left(u_{r}=224 \mathrm{ppm}\right)$.

From the Lamb shift and the 2S-HFS we deduce the proton's rms charge radius

$R_{c h}=0.84087(26)^{\exp }(29)^{\text {th }} \mathrm{fm}=0.84087(39) \mathrm{fm}$

and the Zemach radius

$R_{Z}=1.082(31)^{\exp }(20)^{\text {th }} \mathrm{fm}=1.082(37) \mathrm{fm}$,

respectively [2], using up-to-date theory of QED and nuclear structure effects, recently summarized in Ref. [19].

\subsection{The charge radius of the proton}

The charge radius $R_{c h}$ in Eq. (1) is an order of magnitude more precise than the most recent CODATA-2010 value of $R_{c h}^{\text {CODATA }}=0.8775(51)$ fm [16], but differs by 7 standard deviations $(7 \sigma)$ from it. The CODATA value is obtained from 2 sources, precision spectroscopy in hydrogen $(\mathrm{H})$ and deuterium (D), and elastic electronproton (e-p) scattering. H/D spectroscopy yields $R_{c h}^{\mathrm{H} / \mathrm{D}}=$ 0.8758 (77) fm [16], and e-p scattering recently performed in Mainz gives $R_{c h}^{\text {Mainz }}=0.879(8) \mathrm{fm}$ [17]. Another value recently obtained at Jefferson Lab (JLab) by polarization transfer measurements in e-p scattering is $R_{c h}^{\mathrm{JLab}}=$ 0.875(10) fm [18], again in agreement with the other electronic values, but in disagreement with the muonic value.

One should note, however, that analysis of scattering data using dispersion relations has traditionally yielded 


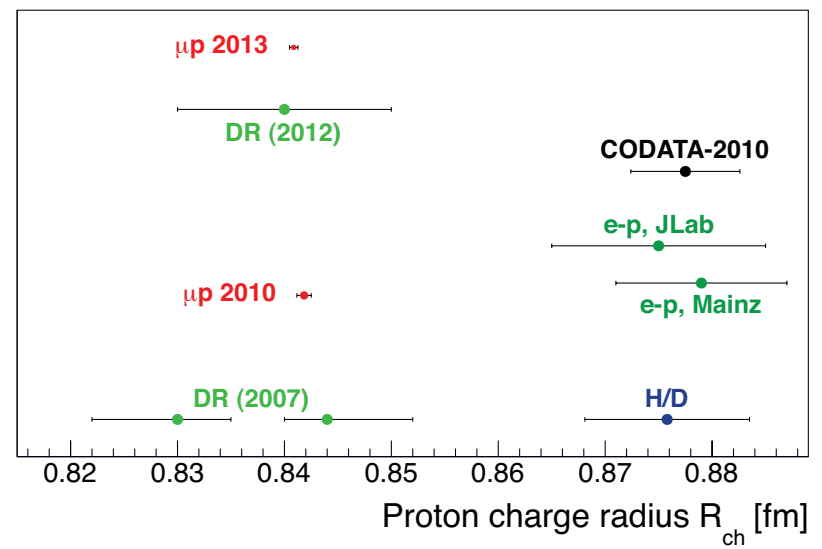

Figure 2 Recent determinations of the rms charge radius of the proton. Muonic hydrogen [1, 2] agrees with dispersion relation (DR) fits $[14,15]$. Spectroscopy in $H$ and $D[16]$ agree with recent elastic e-p scattering from Mainz [17] and JLab [18]. The CODATA-2010 value [16] is mainly based on H/D and Mainz.

a proton charge radius in agreement with the muonic hydrogen value $[14,20]$. Recently, this method has also been applied to the new Mainz data of Ref [17], yielding $R_{c h}^{\mathrm{DR}}=0.84(1) \mathrm{fm}$ [15], again in excellent agreement with the muonic value, albeit with a larger $\chi^{2}$ of the fit to the data. The situation is depicted in Fig. 2

\subsection{The proton radius puzzle}

The $7 \sigma$ discrepancy between the charge radius from muonic hydrogen and the CODATA value has puzzled the physics community for three years now, but no solution has been generally accepted. Briefly, proton charge distributions with bumps or tails have been refuted by e-p scattering data [24]. Molecular effects in muonic hydrogen can also not be responsible for a significant shift of the resonance and therefore of $R_{c h}$ [25]. An unexpectedly large proton polarizability contribution to the Lamb shift in muonic hydrogen $[26,27]$ seems unlikely [28]. Physics beyond the Standard Model needs delicate fine tuning to evade constraints from many precision measurements [29]. For a recent review see Ref. [30].

\subsection{The Zemach radius of the proton}

The Zemach radius of the proton, $R_{Z}$, enters the description of the hyperfine splitting (HFS) in electronic and muonic hydrogen. The HFS originates from the interaction of the lepton and proton magnetic moments. A convolution of the charge and magnetization distribu-

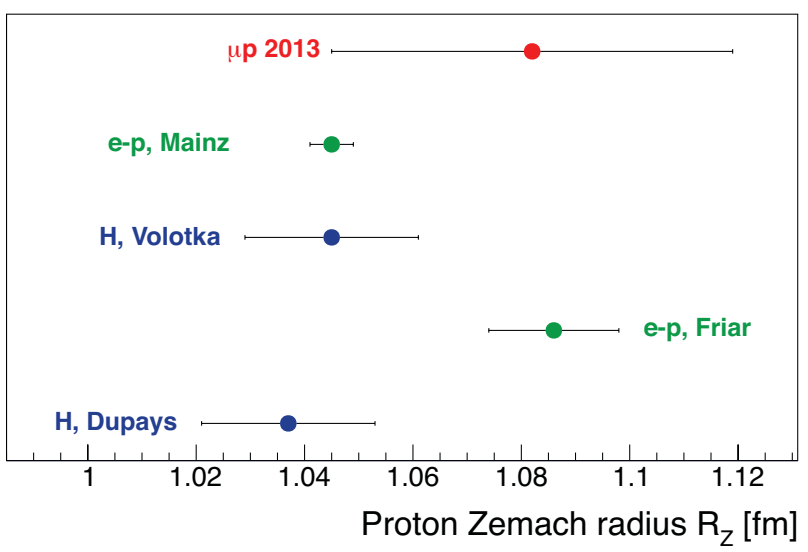

Figure 3 Recent determinations of the proton Zemach radius from $\mathrm{H}[21,22]$, e-p scattering $[23,24]$ and the $2 \mathrm{~S}$ HFS in muonic hydrogen [2].

tions appears as a result of the finite magnetic size of the proton and the distortion of the lepton's wave function due to the finite charge distribution of the proton:

$$
\begin{aligned}
R_{Z} & =\int \mathrm{d}^{3} r r \int \mathrm{d}^{3} r^{\prime} \rho_{\mathrm{ch}}\left(\left|\boldsymbol{r}-\boldsymbol{r}^{\prime}\right|\right) \rho_{\mathrm{mag}}\left(r^{\prime}\right) \\
& =-\frac{4}{\pi} \int_{0}^{\infty} \frac{d q}{q^{2}}\left(G_{E}\left(q^{2}\right) G_{M}\left(q^{2}\right)-1\right) .
\end{aligned}
$$

Here, $\rho_{\text {ch }}(r)$ and $\rho_{\text {mag }}(r)$ are the (normalized) radial charge and magnetization densities of the proton, respectively. $G_{E}$ and $G_{M}$ are the electric and magnetic form factors of the proton.

The Zemach radius from $\mu \mathrm{p}$ is in agreement, but less accurate than the previous values from the HFS in $\mathrm{H}$ $[21,22]$, and e-p scattering $[23,24]$ (see Fig. 3):

- The 2S-HFS of $5 \mathrm{THz}$ was determined from the difference of two Lamb shift transitions at $55 \mathrm{THz}$ and $50 \mathrm{THz}$. This results already in an increase in (relative) uncertainty by more than an order of magnitude.

- The Zemach radius contributes only $0.7 \%$ of the $2 \mathrm{~S}$ HFS in $\mu \mathrm{p}$. In contrast, the charge radius effect on the Lamb shift is as large as $2 \%$.

Muonic hydrogen may provide an improved value of the Zemach radius in the future.

The Zemach radius relates the electric and magnetic form factors of the proton (Eq. (3)). A more accurate value of $R_{Z}$ from muonic hydrogen may therefore be able to resolve the long-standing discrepancy in the measured form factor ratio $G_{E} / G_{M}$ between Rosenbluth and polarization transfer measurements [32]. 


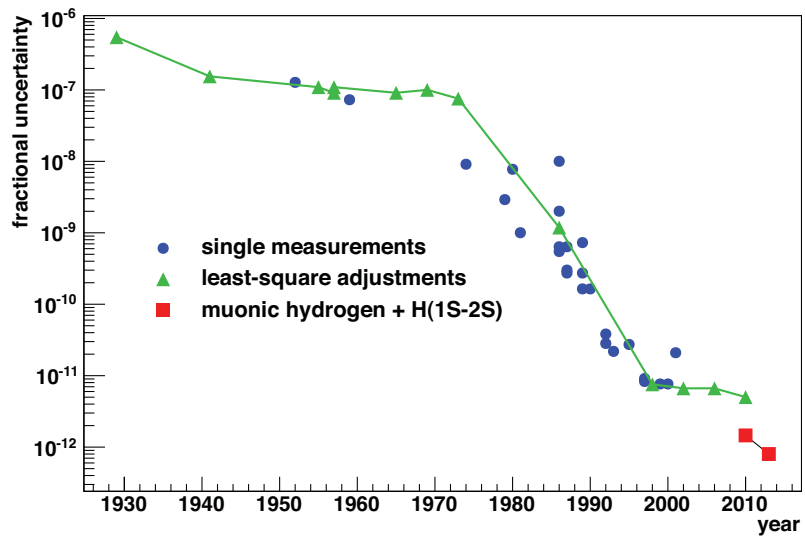

Figure 4 Accuracy of the Rydberg constant over time. The green triangles denote least square adjustments like CODATA [16]. Red squares are the values deduced from the $1 \mathrm{~S}-2 \mathrm{~S}$ transition frequency in hydrogen [31] using the proton charge radius from muonic hydrogen $[1,2]$.

\subsection{Rydberg constant and the charge radius of the deuteron}

Combining the proton charge radius from muonic hydrogen (Eq. (1)) with precision spectroscopy of the 1S2S transition in hydrogen $\mathrm{H}$ [31] and deuterium $\mathrm{D}$ [33] yields new, much more accurate values of the Rydberg constant $R_{\infty}$ and the rms charge radius of the deuteron $R_{c h}(\mathrm{~d})$. Both $R_{\infty}$ and $R_{c h}(\mathrm{~d})$ are $7 \sigma$ away from their respective CODATA-2010 values [16], due to their large correlation $(\sim 1.0)$ with the proton charge radius. Therefore, once the proton radius puzzle is solved, new values of $R_{\infty}$ and $R_{c h}(\mathrm{~d})$ emerge.

The 1S-2S transition in $\mathrm{H}$ has been measured with an accuracy of 4.2 parts in $10^{15}$ [31]. When combined with the muonic $R_{c h}$ and QED theory [16], we obtain

$$
R_{\infty}=3.2898419602495(10)^{\text {radius }}(25)^{\mathrm{QED}} \times 10^{15} \mathrm{~Hz} / \mathrm{c}
$$

which is six times more precise $\left(u_{r}=8 \times 10^{-13}\right)$ than the CODATA-2010 value [16] (Fig. 4). Uncertainties of QED theory in electronic hydrogen $\mathrm{H}$ constitute the main uncertainty of $2.5 \mathrm{kHz} / \mathrm{c}$ in $R_{\infty}$. The uncertainty from the proton charge radius is only $1.0 \mathrm{kHz} / \mathrm{c}$.

The isotope shift of the 1S-2S transition in $\mathrm{H}$ and D is sensitive to the difference of the squared charge radii of the deuteron and the proton. Both experiment [33] and theory [34] have recently been improved by an order of magnitude. Combined with the muonic proton radius
$R_{c h}$ we obtain a deuteron charge radius

$R_{c h}(\mathrm{~d})=2.12771(22) \mathrm{fm}$,

ten times more precise than the CODATA-2010 value $R_{c h}(\mathrm{~d})=2.1424$ (25) fm [16].

The Lamb shift in muonic deuterium, performed by our group, may be able to shed some light on the radius puzzle. Nuclear structure corrections to the Lamb shift and hyperfine splitting in muonic deuterium are however large and difficult to calculate [35].

\section{Outlook}

The proton radius puzzle persists after nearly three years. More data is needed to find its origin. Precision spectroscopy of simple atomic systems may resolve the puzzle $[36,37]$. A measurement of the Lamb shift in muonic helium ions is planned for this year [38].

Acknowledgments. We acknowledge support from the European Research Council (ERC) under Starting Grant \#279765, the MaxPlanck-Society and the Max-Planck-Foundation, the Swiss National Science Foundation (projects 100632 and 138175), the Swiss Academy of Engineering Sciences, the BQR de l'UFR de physique fondamentale et appliquée de l'Université Paris 6, the program PAl Germaine de Staël no. $\sim 07819 \mathrm{NH}$ du ministère des affaires étrangéres France, and the Fundação para a Ciência e a Tecnologia (Portugal) and FEDER (project PTDC/-FIS/-82006/-2006 and grant SFRH/BPD/46611/2008). P. I. acknowledges support by the ExtreMe Matter Institute, Helmholtz Alliance HA216/EMMI.

Key words. Muonic hydrogen, Proton radius, Charge radius, Zemach radius, Rydberg constant, Proton radius puzzle.

\section{References}

[1] R. Pohl, A. Antognini, F. Nez, F. D. Amaro, F. Biraben, J. M. R. Cardoso, D. S. Covita, A. Dax, S. Dhawan, L. M. P. Fernandes, A. Giesen, T. Graf, T. W. Hänsch, P. Indelicato, L. Julien, C. Y. Kao, P. Knowles, E. O. L. Bigot, Y. W. Liu, J. A. M. Lopes, L. Ludhova, C. M. B. Monteiro, F. Mulhauser, T. Nebel, P. Rabinowitz, J. M. F. dos Santos, L. A. Schaller, K. Schuhmann, C. Schwob, D. Taqqu, J. F. C. A. Veloso, and F. Kottmann, Nature 466, 213 (2010).

[2] A. Antognini, F. Nez, K. Schuhmann, F. D. Amaro, F. Biraben, J. M. R. Cardoso, D. S. Covita, A. Dax, S. Dhawan, M. Diepold, L. M. P. Fernandes, A. Giesen, T. Graf, A. L. Gouvea, T. W. Hänsch, P. Indelicato, L. Julien, C. Y. Kao, P. Knowles, F. Kottmann, E. O. L. Bigot, Y. W. Liu, J. A. M. Lopes, L. Ludhova, C. M. B. Monteiro, F. Mulhauser, T. Nebel, P. Rabinowitz, J. M. F. dos Santos, L. A. Schaller, C. Schwob, D. Taqqu, J. F. C. A. 
Veloso, J. Vogelsang, and R. Pohl, Science 339, 417 (2013).

[3] R. Pohl, A. Antognini, F. Amaro, F. Biraben, J. Cardoso, C. Conde, A. Dax, S. Dhawan, L. Fernandes, T. Hansch, F. Hartmann, V. Hughes, O. Huot, P. Indelicato, L. Julien, P. Knowles, F. Kottmann, Y. W. Liu, L. Ludhova, C. Monteiro, F. Mulhauser, F. Nez, P. Rabinowitz, J. dos Santos, L. Schaller, C. Schwob, D. Taqqu, and J. Veloso, Can. J. Phys. 83(4), 339-349 (2005).

[4] F. Kottmann, H. Daniel, F. J. Hartmann, P. Hauser, C. Maierl, V. E. Markushin, M. Mühlbauer, C. Petitjean, R. Pohl, W. Schott, and D. Taqqu, Hyp. Interact. 119, 3 (1999).

[5] T. S. Jensen and V. E. Markushin, Eur. Phys. J. D 21 (October), 261-270 (2002).

[6] T. S. Jensen and V. E. Markushin, Eur. Phys. J. D 21 (October), 271-283 (2002).

[7] V. P. Popov and V. N. Pomerantsev, Phys. Rev. A 83, 032516 (2011).

[8] R. Pohl, Hyp. Interact. 193, 115-120 (2009).

[9] R. Pohl, H. Daniel, F. J. Hartmann, P. Hauser, Y. W. Liu, F. Kottmann, C. Maierl, V. E. Markushin, M. Mühlbauer, C. Petitjean, W. Schott, and D. Taqqu, Hyp. Interact. 138, 35-40 (2001).

[10] R. Pohl, H. Daniel, F. J. Hartmann, P. Hauser, F. Kottmann, V. E. Markushin, M. Muhlbauer, C. Petitjean, W. Schott, D. Taqqu, and P. WojciechowskiGrosshauser, Phys. Rev. Lett. 97, 193402 (2006).

[11] L. Ludhova, F. D. Amaro, A. Antognini, F. Biraben, J. M. R. Cardoso, C. A. N. Conde, D. S. Covita, A. Dax, S. Dhawan, L. M. P. Fernandes, T. W. Hänsch, V. W. Hughes, O. Huot, P. Indelicato, L. Julien, P. E. Knowles, F. Kottmann, J. A. M. Lopes, Y. W. Liu, C. M. B. Monteiro, F. Mulhauser, F. Nez, R. Pohl, P. Rabinowitz, J. M. F. dos Santos, L. A. Schaller, D. Taqqu, and J. F. C. A. Veloso, Nucl. Inst. Meth. A 540(1), 169-179 (2005).

[12] A. Antognini, F. D. Amaro, F. Biraben, J. M. R. Cardoso, C. A. N. Conde, D. S. Covita, A. Dax, S. Dhawan, L. M. P. Fernandes, T. W. Hänsch, V. . Hughes, O. Huot, P. Indelicato, L. Julien, P. E. Knowles, F. Kottmann, Y. W. Liu, J. A. M. Lopes, L. Ludhova, C. M. B. Monteiro, F. Mulhauser, F. Nez, B. N. Perry, R. Pohl, P. Rabinowitz, J. M. F. dos Santos, L. A. Schaller, C. Schwob, D. Taqqu, and J. F. C. A. Veloso, Opt. Comm. 253(4-6), 362-374 (2005).

[13] A. Antognini, K. Schuhmann, F. D. Amaro, F. Biraben, A. Dax, A. Giesen, T. Graf, T. W. Hänsch, P. Indelicato, L. Julien, K. Cheng-Yang, P. E. Knowles, F. Kottmann, E. Le Bigot, Y. W. Liu, L. Ludhova, N. Moschüring, F. Mulhauser, T. Nebel, F. Nez, P. Rabinowitz, C. Schwob, D. Taqqu, and R. Pohl, IEEE J. Quant. Electr. 45(8), 9931005 (2009).

[14] M. A. Belushkin, H. W. Hammer, and U. G. Meissner, Phys. Rev. C 75, 035202 (2007).
[15] I. Lorenz, H. W. Hammer, and U. G. Meissner, Eur. Phys. J. A 48, 151 (2012).

[16] P. J. Mohr, B. N. Taylor, and D. B. Newell, Rev. Mod. Phys. 84, 1527 (2012).

[17] J. C. Bernauer, P. Aschenbach, C. Ayerbe Gayoso, R. Böhm, D. Bosnar, L. Debenjak, M. O. Distler, L. Doria, A. Esser, et al., Phys. Rev. Lett. 105, 242001 (2010).

[18] X. Zhan, K. Allada, D. S. Armstrong, J. Arrington, W. Bertozzi, W. Boeglin, et al., Phys. Lett. B 705, 59 (2011).

[19] A. Antognini, F. Kottmann, F. Biraben, P. Indelicato, F. Nez, and R. Pohl, Ann. Phys. 331, 127 (2013).

[20] C. Adamuscin, S. Dubnicka, and A. Z. Dubnickova, Prog. Part. Nucl. Phys. 67, 479 (2012).

[21] A. Dupays, A. Beswick, B. Lepetit, C. Rizzo, and D. Bakalov, Phys. Rev. A 68, 052503 (2003).

[22] A. V. Volotka, V. M. Shabaev, G. Plunien, and G. Soff, Eur. Phys. J. D 33, 23-27 (2005).

[23] J. Friar and I. Sick, Phys. Lett. B 579, 285 (2004).

[24] M. O. Distler, J. C. Bernauer, and T. Walcher, Phys. Lett. B 696, 343 (2011).

[25] J. P. Karr and L. Hilico, Phys. Rev. Lett. 109, 103401 (2012).

[26] G. A. Miller, A. W. Thomas, J. D. Carroll, and J. Rafelski, Phys. Rev. A 84, 020101(R) (2011).

[27] R. J. Hill and G. Paz, Phys. Rev. Lett. 107, 160402 (2011).

[28] M. C. Birse and J. A. McGovern, Eur. Phys. J. A 48, 120 (2012).

[29] C. E. Carlson and B. C. Rislow, Phys. Rev. D 86, 035013 (2012).

[30] R. Pohl, R. Gilman, G. A. Miller, and K. Pachucki, DOI: 10.1146/annurev-nucl-102212-170627.

[31] C. G. Parthey, A. Matveev, J. Alnis, B. Bernhard, A. Beyer, R. Holzwarth, A. Maistrou, R. Pohl, K. Predehl, T. Udem, T. Wilken, N. Kolachevsky, M. Abgrall, D. Rovera, C. Salomon, P. Laurent, and T. W. Hänsch, Phys. Rev. Lett. 107, 203001 (2011).

[32] S. J. Brodksy, C. E. Carlson, J. R. Hiller, and D. S. Hwang, Phys. Rev. Lett. 94, 022001 (2005).

[33] C. G. Parthey, A. Matveev, J. Alnis, R. Pohl, T. Udem, U. D. Jentschura, N. Kolachevsky, and T. W. Hänsch, Phys. Rev. Lett. 104(June), 233001 (2010).

[34] U. D. Jentschura, A. Matveev, C. G. Parthey, J. Alnis, R. Pohl, T. Udem, N. Kolachevksy, and T. W. Hänsch, Phys. Rev. A 83, 042505 (2011).

[35] K. Pachucki, Phys. Rev. Lett. 106, 192007 (2011).

[36] A. Beyer, et al., this issue.

[37] E. Peters, et al., this issue.

[38] A. Antognini, F. Nez, F. D. Amaro, F. Biraben, J. M. R. Cardoso, D. S. Covita, A. Dax, S. Dhawan, L. M. P. Fernandes, A. Giesen, T. Graf, T. W. Hänsch, P. Indelicato, L. Julien, C. Y. Kao, P. Knowles, F. Kottmann, E. O. L. Bigot, Y. W. Liu, J. A. M. Lopes, L. Ludhova, C. M. B. Monteiro, F. Mulhauser, T. Nebel, P. Rabinowitz, J. M. F. dos Santos, L. A. Schaller, K. Schuhmann, C. Schwob, D. Taqqu, J. F. C. A. Veloso, and R. Pohl, Can. J. Phys. 89, 47 (2010). 